\title{
Flebotomíneos do Estado de Tocantins, Brasil (Diptera: Psychodidae)
}

\author{
Phlebotomine sand flies in the State of Tocantins, Brazil (Diptera: Psychodidae) \\ José Dilermando Andrade Filho1, Marcela Bortolini Valente ${ }^{1,3}$, Welton A. de Andrade ${ }^{2}$, \\ Reginaldo Peçanha Brazil' e Alda Lima Falcão ${ }^{1}$
}

\begin{abstract}
Resumo Nos anos de 1997 e 1998 realizaram-se capturas esporádicas de flebotomíneos nos municípios de Paraíso de Tocantins, Monte do Carmo, Porto Nacional e Monte Santo, todos localizados no estado de Tocantins, região norte do Brasil, com o intuito de conhecer a fauna flebotomínica da região. No município de Monte Santo utilizou-se isca humana e nos demais armadilhas luminosas CDC. Os ecótopos utilizados foram: peridomicílio, intradomicílio, mata e pomar. Foram capturados 2.677 flebotomíneos, pertencentes a 32 espécies. As mais freqüentes foram Lutzomyia whitmani, Lutzomyia longipalpis, Lutzomyia carmelinoi, Lutzomyia evandroi, Lutzomyia longipennis e Lutzomyia antunesi. As capturas na mata apresentaram maior diversidade de espécies, enquanto no peridomicílio houve maior número de exemplares capturados. Foram capturadas neste estado várias espécies vetoras em outras regiões do Brasil.
\end{abstract}

Palavras-chaves: Diptera. Phlebotominae. Lutzomyia. Tocantins.

Abstract Between 1997-1998, the authors carried out sporadic collection of sand flies in the municipalities of Paraíso de Tocantins, Monte do Carmo, Porto Nacional and Monte Santo all in the Tocantins State of northern Brazil. Human bait was used in Monte Santo and a battery operated light trap in other municipalities. The ecotypes chosen for the traps were in the peridomiciles, inside the houses, in the forest and the orchard. We identified 2,677 sand flies, belonging to 32 species. The most abundant species of sand flies were Lutzomyia whitmani, Lutzomyia longipalpis, Lutzomyia carmelinoi, Lutzomyia evandroi, Lutzomyia longipennis and Lutzomyia antunesi. Collections from the forest showed greater diversity of species, while the largest number of sand flies were caught around the houses. Several species known or suspected to be vectors of Leishmania in other regions of Brazil were captured.

Key-words: Diptera. Phlebotominae. Lutzomyia. Tocantins.

O conhecimento da fauna de flebotomíneos mostrou-se de grande importância devido à capacidade desses insetos de transmitir patógenos como Leishmania, Bartonella bacilliformis e algumas arboviroses. O hábito hematófago restringe-se às fêmeas, e o horário de maior atividade se dá ao crepúsculo vespertino, estendendo-se durante a noite.

$\mathrm{Na}$ região neotropical os flebotomíneos encontram-se distribuídos em todos os países, sendo que algumas espécies possuem ampla distribuição geográfica, abrangendo vários países sulamericanos. No Brasil, diversos trabalhos vêm sendo realizados sobre distribuição geográfica, sazonalidade, ecologia e epidemiologia destes insetos ${ }^{481121}$, mas, em algumas regiões do país, pouco se conhece sobre a fauna dos flebotomíneos.

O Estado de Tocantins, criado pela constituição de 1988, apresenta uma área de $278.420,7 \mathrm{~km}^{2}$, e uma população de 920.116 habitantes, distribuídos por 123 municípios agrupados em oito microrregiões. Neste estado, têm sido relatados casos esporádicos de leishmaniose tegumentar e visceral.

Devido ao pouco conhecimento sobre os flebotomíneos do Estado de Tocantins, procurouse fazer um estudo da fauna destes dípteros existentes na região.

\footnotetext{
1. Laboratório de Leishmanioses do Centro de Pesquisas René Rachou da Fundação Oswaldo Cruz, Belo Horizonte, MG. 2. Distrito Sanitário de Porto Nacional, Fundação Nacional de Saúde, Porto Nacional, TO. 3. Bolsista do PIBIC

Endereço para correspondência: Dr. José Dilermando Andrade Filho. Centro de Pesquisas René Rachou/FIOCRUZ. Av. Augusto de Lima 1715, Barro Preto, 30190-002 Belo Horizonte, MG, Brasil.

Tel. 55313295 -3566, Fax $55313295-3115$.

e-mail:jandrade@cpqrr.fiocruz.br

Recebido para publicação em 07/02/2000
} 


\section{MATERIAL E MÉTODOS}

O Estado de Tocantins situa-se na região norte e ocupa uma zona de transição entre o Planalto Central brasileiro, de clima mais seco e vegetação dominada pelo cerrado, e a Planície Amazônica, úmida na maior parte do ano e coberta por floresta equatorial. O relevo é em grande parte formado por planaltos sedimentares, de altitude média no centro-sul, e com chapadões mais altos na parte oriental. Nesta região está a planície da bacia do rio Araguaia, periodicamente inundada. Na região central, numa faixa que se estende na direção sul-norte, está a planície da bacia do Tocantins. Entre os dois grandes sistemas hidrográficos, encontram-se as chapadas e chapadões.

Área de estudo: (Figura 1) O município de Monte do Carmo tem uma população de 5.429 habitantes, uma área de $3.359,7 \mathrm{~km}^{2}$ e altitude máxima de 295 metros. Sua latitude é de $10^{\circ} 45^{\prime} 48^{\prime \prime}$ S e longitude de $48^{\circ} 06^{\prime} 32^{\prime \prime}$ W. A vegetação predominante é o cerrado, sendo bem conservadas as matas ciliares. A principal atividade profissional é a agropecuária e o turismo. Entre 1996 a maio de 2000 foram registrados para este município 7 casos de leishmaniose visceral e 40 casos de leishmaniose tegumentar.

Monte Santo apresenta uma população de 1.064 habitantes e área de $1.082,7 \mathrm{~km}^{2}$. A agropecuária e a mineração são as principais fontes de renda do município. O cerrado predomina e apenas um caso de leishmaniose tegumentar foi registrado entre 1996 e maio de 2000. Não há referência para as coordenadas geográficas deste município.

A vegetação de cerrado predomina em Paraíso do Tocantins, município que tem uma população de 35.884 habitantes dentro de uma área de $1.331 \mathrm{~km}^{2}$ e altitude máxima de $387 \mathrm{~m}$. Sua latitude é de $10^{\circ} 10^{\prime} 34^{\prime \prime} \mathrm{S}$ e a longitude é de $48^{\circ} 52^{\prime} 00^{\prime \prime} \mathrm{W}$. A agropecuária e pequenas indústrias predominam como as principais atividades do município. A leishmaniose visceral acometeu 72 pessoas enquanto a leishmaniose tegumentar registrou 74 casos entre 1996 e maio de 2000, para ambas.
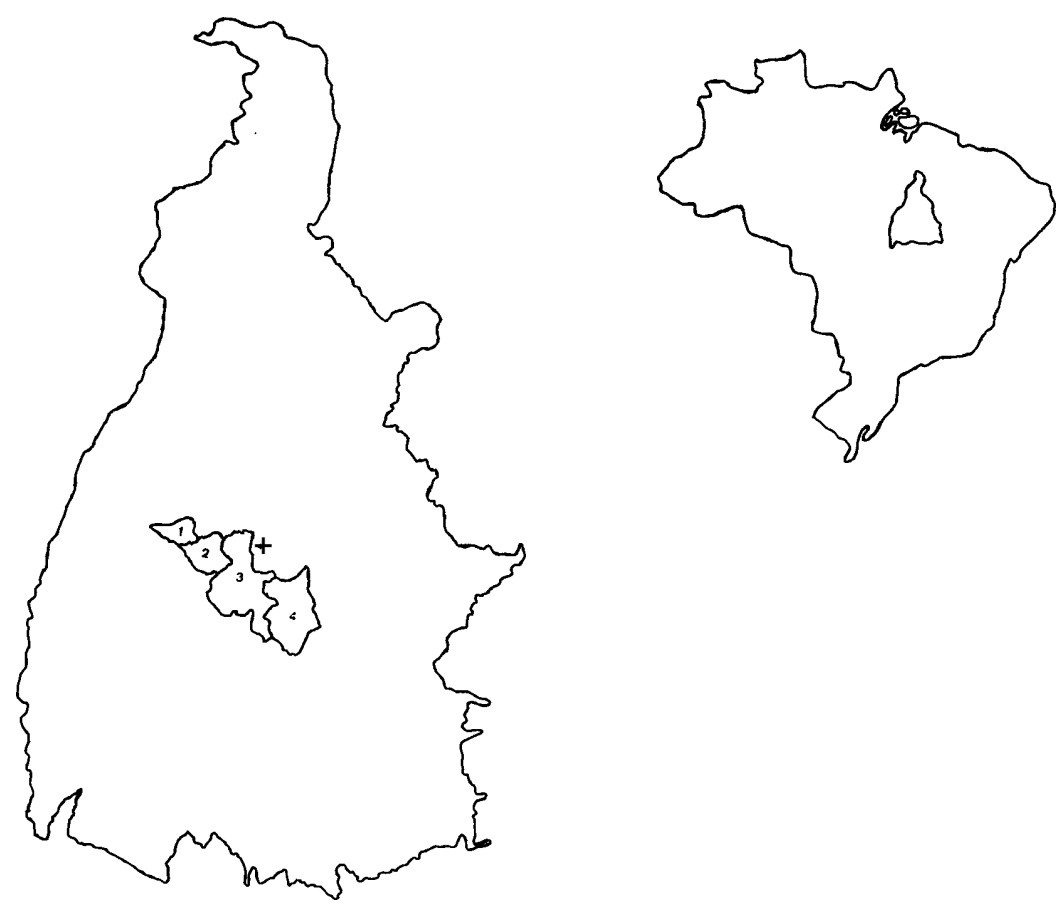

1. Monte Santo, 2. Paraiso do Tocantins, 3. Porto Nacional, 4. Monte do Carmo, += Palmas.

Figura 1 - Mapa do Brasil mostrando a loçalização do Estado do Tocantins e Mapa do Estado de Tocantins com os quatro municípios trabalhados e a localizaçâo da Capital Palmas. 
Porto Nacional representa um dos principais municípios do Estado de Tocantins. Sua população é de 43.035 habitantes e a área do município é de 4.464,1 $\mathrm{km}^{2}$. A altitude máxima é de 212 metros e está situado geograficamente entre os paralelos $10^{\circ} 42^{\prime} 29^{\prime \prime}$ de latitude Sul e $48^{\circ} 25^{\prime} 02^{\prime \prime}$ de longitude Oeste. A agropecuária e pequenas industrias são as principais atividades profissionais. Registrou-se neste município, entre 1996 e maio de 2000, 71 casos de leishmaniose visceral e 74 casos de leishmaniose tegumentar

Captura dos flebotomíneos: Os flebotomíneos foram obtidos através de capturas realizadas no mês de dezembro de 1997, em 19 regiões de quatro municípios do Estado de Tocantins: Porto Nacional, Paraíso do Tocantins, Monte do Carmo e Monte Santo. No município de Porto Nacional realizaram-se sete capturas, sendo cinco na cidade (quatro em galinheiros, uma em um galpão que serve de abrigo para animais domésticos), uma na mata (fazenda Mercés) e outra em um bananal localizado no povoado de Pinheirinho. Em Monte do Carmo, foram selecionados cinco locais de captura dentro da fazenda Córrego da Cruz, sendo três na mata, um em galinheiro e o último em um curral. No terceiro município, Paraíso do Tocantins, as capturas foram feitas em cinco galinheiros localizados dentro da cidade. Nestes municípios utilizou-se armadilha luminosa do tipo CDC ${ }^{22}$, que foram expostas entre 16:00 e 17:00 horas e recolhidas no crepúsculo matutino. Em Monte Santo, foram feitas duas capturas com isca humana na mata, às margens de um córrego, na fazenda Matona.

Em setembro de 1998, realizou-se mais 19 capturas em Porto Nacional, duas em galinheiros na cidade, e o restante nas fazendas Mutum (duas em pomar e uma em galinheiro), Jacozinho (uma na mata, uma no intradomicílio e outra em um curral), São Pedro (duas na mata, duas em galinheiro e uma no intradomicílio), Mato Escuro (uma em galinheiro e outra na mata), Mata Grande (uma em galinheiro, uma em pomar e outra em um curral) e Francisquinha (uma no galinheiro). Na fazenda Córrego da Cruz, município de Monte do Carmo, efetuou-se mais cinco capturas, sendo duas em galinheiro, duas em mata e uma em um pomar.

Os flebotomíneos capturados foram fixados em álcool $70^{\circ}$ e trazidos ao laboratório onde foram preparados e montados entre lâmina e lamínula em líquido de Berlese e Bálsamo do Canadá. A identificação foi feita de acordo com Young \& Duncan ${ }^{26}$, sendo que para a identificação das espécies do grupo oswaldoi foi utilizado o trabalho de Dias et $\mathrm{al}^{10}$. Após a montagem e a identificação os insetos foram numerados e incorporados à coleção de flebotomíneos do Centro de Pesquisas René Rachou/FIOCRUZ, sob os números 71.533 a 72.266 e 72.824 a 74.767 .

Os ecótopos utilizados para comparação foram o peridomicílio, que compreendia abrigos de animais domésticos e árvores isoladas ao redor da casa, intradomicílio, mata e pomar.

Para comparar os dados das capturas realizadas nos cinco municípios e entre os ecótopos, foram calculadas a média aritmética simples, dividindo-se o número de exemplares de uma espécie pelo número de capturas no município ou no ecótopo.

\section{RESULTADOS}

Em 43 capturas, foram obtidos 2.677 flebotomíneos, pertencentes a 32 espécies; duas destas espécies pertencem ao gênero Brumptomyia França \& Parrot e as demais ao gênero Lutzomyia França (Tabela 1). As espécies deste último gênero estavam divididas entre nove subgêneros e cinco grupos. Uma espécie, Lutzomyia sp 01 foi considerada espécie isolada. As espécies capturadas foram: Lutzomyia (Nyssomyia) whitmani (Antunes \& Coutinho) (53\%) Lutzomyia (Lutzomyia) longipalpis (Lutz \& Neiva) (25,2\%), Lutzomyia carmelinoi Ryan Fraiha Lainson \& Shaw $(6,9 \%)$, Lutzomyia evandroi (Costa Lima \& Antunes) $(3,4 \%)$, Lutzomyia longipennis (Barretto) $(1,8 \%)$, Lutzomyia (Nyssomyia) antunesi (Coutinho) (1,6\%), Lutzomyia sp. 01 (1,1\%), Lutzomyia (Sciopemyia) sordellii (Shannon \& Del Ponte) (1,1\%), Lutzomyia sallesi (Galvão \& Coutinho) $(0,9 \%)$, Lutzomyia (Evandromyia) bourrouli (Barretto \& Coutinho) $(0,8 \%)$, Lutzomyia peresi (Mangabeira) (0,7\%), Lutzomyia termitophila Martins Falcão \& Silva (0,5\%), Lutzomyia aragaoi (Costa Lima) (0,3\%), Lutzomyia hermanlenti Martins Silva \& Falcão (0,3\%), Lutzomyia goiana Martins Falcão \& Silva (0,3\%), Lutzomyia lenti (Mangabeira) $(0,3 \%)$, Lutzomyia (Nyssomyia) flaviscutellata
(Mangabeira) (0,2\%), Lutzomyia (Micropygomyia) micropyga (Mangabeira) (0,2\%), Lutzomyia saulensis (Floch \& Abonnenc) $(0,2 \%)$, Lutzomyia (Psathyromyia) sp. $(0,2 \%)$ Lutzomyia (Psathyromyia) shannoni (Dyar) $(0,1 \%)$, Lutzomyia bacula Martins Falcão \& Silva $(0,1 \%)$, Lutzomyia (Pintomyia) christenseni Young \& Duncan $(0,1 \%)$, Lutzomyia oswaldoi (Mangabeira) $(0,1 \%)$, Lutzomyia (Psathyromyia) abonnenci (Floch \& Chassignet) (<0,1\%), Lutzomyia (Psathyromyia) campbelli (Damasceno Causey \& Arouck) $(<0,1 \%)$, Lutzomyia (Psychodopygus) claustrei Abonnenc Léger \& Fauran $(<0,1 \%)$, Lutzomyia rorotaensis (Floch \& Abonnenc) (<0,1\%), Lutzomyia (Viannamyia) tuberculata (Mangabeira) $(<0,1 \%)$, Lutzomyia walkeri (Newstead) $(<0,1 \%)$, Brumptomyia avellari (Costa Lima) $(0,1 \%)$, Brumptomyia brumpti (Larrousse) $(0,1 \%)$.

O município de Porto Nacional apresentou a maior diversidade de espécies (26), enquanto Monte do Carmo mostrou o maior rendimento. Os piores resultados foram em Monte Santo, onde o número de flebotomíneos amostrados foi pequeno.

O peridomicílio foi o ecótopo onde se obteve maior rendimento de exemplares capturados, 64 flebotomíneos por coleta e a mata apresentou maior 
Tabela 1 - Flebotomíneos capturados em quatro municípios do Estado de Tocantins, Brasil, em dezembro de 1997 e setembro de 1998.

\begin{tabular}{|c|c|c|c|c|c|}
\hline \multirow[b]{2}{*}{ Espécies } & \multicolumn{5}{|c|}{ Município } \\
\hline & Porto Nacional & Paraíso do Tocantins & Monte do Carmo & Monte Santo & Total \\
\hline L. whitmani & 476 & 53 & 889 & - & 1.418 \\
\hline L. longipalpis & 290 & 345 & 40 & 1 & 676 \\
\hline L. carmelinoi & 37 & 7 & 142 & - & 186 \\
\hline L. evandroi & 27 & 18 & 47 & - & 92 \\
\hline L. longipennis & 45 & 1 & 1 & 1 & 48 \\
\hline L. antunesi & 25 & 2 & 8 & 9 & 44 \\
\hline Lutzomyia sp 01 & 30 & - & - & - & 30 \\
\hline L. sordellii & 15 & 1 & 13 & - & 29 \\
\hline L. sallesi & 21 & - & 2 & 2 & 25 \\
\hline L. bourrouli & 21 & - & - & - & 21 \\
\hline L. peresi & 16 & 2 & - & - & 18 \\
\hline L. termitophila & 8 & 2 & 4 & - & 14 \\
\hline L. aragaoi & 1 & - & 7 & - & 8 \\
\hline L. hermanlenti & 2 & - & 6 & - & 8 \\
\hline L. goiana & 5 & 1 & 1 & - & 7 \\
\hline L. lenti & 4 & - & 3 & - & 7 \\
\hline L. flaviscutellata & 2 & - & 4 & - & 6 \\
\hline L. micropyga & 1 & - & 5 & - & 6 \\
\hline L. saulensis & 3 & - & 2 & 1 & 6 \\
\hline L.(Psathyromyia) sp. & 4 & - & - & 1 & 5 \\
\hline L. shannoni & 2 & - & 2 & - & 4 \\
\hline L. bacula & - & - & 2 & 1 & 3 \\
\hline L. christenseni & - & - & 3 & - & 3 \\
\hline L. oswaldoi & 3 & - & - & - & 3 \\
\hline L. abonnenci & - & 1 & - & - & 1 \\
\hline L. campbelli & 1 & - & - & - & 1 \\
\hline L. claustrei & - & - & 1 & - & 1 \\
\hline L. rorotaensis & - & - & 1 & - & 1 \\
\hline L. tuberculata & - & - & 1 & - & 1 \\
\hline L. walkeri & 1 & - & - & - & 1 \\
\hline B. avellari & 2 & - & - & - & 2 \\
\hline B. brumpti & 1 & - & 1 & - & 2 \\
\hline Total & 1.043 & 433 & 1.185 & 16 & 2.677 \\
\hline
\end{tabular}

L. = Lutzomyia; B. = Brumptomyia

biodiversidade destes insetos, sendo capturadas 28 das 32 espécies neste ecótopo. (Tabela 2).

L. whitmani, L. carmelinoi e L. evandroi predominaram no município de Monte do Carmo, enquanto L. longipalpis, L. longipennis, Lutzomyia sp. 01, L. sallesi e L. bourrouli foram mais capturadas em Porto Nacional, sendo que esta última só foi capturada neste município. L. antunesi teve freqüência semelhante nos quatro municípios, assim como L. sordellii, sendo que esta não foi capturada em Monte Santo.
L. whitmani, L. Iongipalpis, L. carmelinoi e $L$. evandroi apareceram com maior incidência no peridomicílio, enquanto L. longipennis, L. sordellii e L. sallesi foram capturadas em maior quantidade na mata. L. antunesi e L. bourrouli tiveram freqüência semelhante no peridomicílio e na mata. Lutzomyia sp. 01 foi capturada tanto no peridomicílio quanto no pomar. No intradomicílio predominaram as espécies L. whitmani e L. longipalpis. 
Tabela 2 - Total de flebotomíneos capturados em cinco ecótopos no Estado de Tocantins, Brasil, em dezembro de 1997 e setembro de 1998. Em parênteses a média destes insetos por captura.

\begin{tabular}{|c|c|c|c|c|c|}
\hline \multirow{3}{*}{ Espécies } & \multicolumn{4}{|c|}{ Ecótopo } & \multirow{3}{*}{ Total } \\
\hline & Peridomicílio & Intradomicílio & Mata & Pomar & \\
\hline & № & № & № & № & \\
\hline L. whitmani & $1.268(55,1)$ & $10(5,0)$ & $107(9,6)$ & $33(4,7)$ & 1.418 \\
\hline L. longipalpis & $654(28,4)$ & $9(4,5)$ & $7(0,6)$ & $6(0,9)$ & 676 \\
\hline L. carmelinoi & $157(6,8)$ & $1(0,5)$ & $16(1,5)$ & $12(1,7)$ & 186 \\
\hline L. evandroi & $84(3,7)$ & $1(0,5)$ & $4(0,4)$ & $3(0,4)$ & 92 \\
\hline L. longipennis & $3(0,2)$ & $2(1,0)$ & $41(3,7)$ & $2(0,3)$ & 48 \\
\hline L. antunesi & $28(1,5)$ & - & $16(1,5)$ & - & 44 \\
\hline Lutzomyia sp 01 & $10(2,5)$ & - & - & $20(2,9)$ & 30 \\
\hline L. sordellii & $6(0,3)$ & $1(0,5)$ & $21(1,9)$ & $1(0,1)$ & 29 \\
\hline L. sallesi & $6(0,3)$ & - & $17(1,6)$ & $2(0,3)$ & 25 \\
\hline L. bourrouli & $12(0,6)$ & - & $9(0,8)$ & - & 21 \\
\hline L. peresi & $14(0,6)$ & - & $3(0,3)$ & $1(0,1)$ & 18 \\
\hline L. termitophila & $8(0,4)$ & $1(0,5)$ & $4(0,4)$ & $1(0,1)$ & 14 \\
\hline L. aragaoi & - & - & $8(0,7)$ & - & 8 \\
\hline L. hermanlenti & $1(0,3)$ & - & $6(0,6)$ & $1(0,1)$ & 8 \\
\hline L. goiana & $3(0,1)$ & - & $1(0,1)$ & $3(0,4)$ & 7 \\
\hline L. lenti & $4(0,2)$ & - & $2(0,2)$ & $1(0,1)$ & 7 \\
\hline L. flaviscutellata & $1(0,1)$ & - & $5(0,5)$ & - & 6 \\
\hline L. micropyga & $1(0,3)$ & - & $4(0,4)$ & $1(0,1)$ & 6 \\
\hline L. saulensis & $1(0,1)$ & - & $4(0,4)$ & $1(0,1)$ & 6 \\
\hline L. (Psathyromyia) sp & $4(0,2)$ & - & $1(0,1)$ & - & 5 \\
\hline L. shannoni & $4(0,2)$ & - & - & - & 4 \\
\hline L. bacula & - & - & $3(0,3)$ & - & 3 \\
\hline L. christenseni & - & - & $3(0,3)$ & - & 3 \\
\hline L. oswaldoi & $1(0,1)$ & - & $1(0,1)$ & $1(0,1)$ & 3 \\
\hline L. abonenci & $1(0,1)$ & - & - & - & 1 \\
\hline L. campbelli & - & $1(0,5)$ & - & - & 1 \\
\hline L. claustrei & - & - & $1(0,1)$ & - & 1 \\
\hline$L$ rorotaensis & - & - & $1(0,1)$ & - & 1 \\
\hline L. tuberculata & - & - & $1(0,1)$ & - & 1 \\
\hline L. walkeri & - & - & $1(0,1)$ & - & 1 \\
\hline B. avellari & - & - & $2(0,2)$ & - & 2 \\
\hline B. brumpti & $1(0,1)$ & - & $1(0,1)$ & - & 2 \\
\hline Total & 2.272 & 26 & 290 & 89 & 2.677 \\
\hline
\end{tabular}

L. = Lutzomyia; B. = Brumptomyia

\section{DISCUSSÃO}

A presença de 32 espécies encontradas nos municípios trabalhados demonstra a grande diversidade da fauna da região. O Estado de Tocantins, por estar situado geograficamente na região centro-norte do país, apresenta algumas áreas de transição, tanto vegetacional quanto climática, entre o cerrado e a
Floresta Amazônica e, com novas investigações deverá apresentar um número maior de espécies.

A maior diversidade de espécies ocorreu na mata, entretanto, o maior número de exemplares capturados ocorreu no peridomicílio, o que mostra o grau de adaptação que os flebotomíneos vêm sofrendo com as 
crescentes alterações antrópicas, sendo que aquelas espécies que só ocorreram na mata foram capturas em uma freqüência muito baixa.

As capturas com isca humana apresentam maior número de exemplares quando associadas à armadilha de Shannon ${ }^{20} \mathrm{com}$ isca luminosa. Esse fato explica 0 baixo índice de flebotomíneos capturados em Monte Santo, onde utilizou-se apenas isca humana. Dos 16 exemplares capturados, L. antunesi predominou. Este flebotomíneo mostrou-se altamente antropofílico, já tendo sido encontrado exemplares naturalmente infectados com flagelados no Brasil ${ }^{19}$. As demais espécies foram pouco freqüentes e com exceção de L. longipalpis não há registro de antropofilia para as outras, mas a coleta de algumas delas, como L. longipennis, pode ser acidental.

L. whitmani foi a espécie mais freqüente neste estudo e a mais encontrada no peridomicílio com cerca de 55 flebotomíneos por captura. Essa espécie apresenta ampla distribuição geográfica, representando grande adaptação aos diferentes nichos ecológicos e acarretando mudanças comportamentais nas diversas populações. L. whitmani habita em áreas de floresta primária na região do Amazonas, regiões de florestas remanescentes e peridomicílio no nordeste e sudeste ${ }^{16}$. Vários autores já a capturaram em maior número no peridomicílio, especialmente no sudeste do Brasil ${ }^{314}$, porém, em trabalho realizado no Maranhão, região nordeste, L. whitmani foi capturada com a mesma freqüência, tanto no peridomicílio quanto na mata ${ }^{17}$. $\mathrm{Na}$ região sudoeste do Estado de São Paulo ela foi mais encontrada no ambiente florestal ${ }^{9}$. Esta espécie revelase bastante antropofílica na região nordeste e sudeste $^{57}$, sendo umas das principais vetoras, ou suspeita de transmitir, o agente etiológico leishmaniose tegumentar em várias regiões do Brasil ${ }^{1523}$. Destaca-se que neste trabalho foram capturados dez exemplares no intradomicílio nas duas coletas realizadas, o que pode ser uma tendência a domiciliação dessa espécie.

A espécie Lutzomyia longipalpis representa a principal vetora do agente da leishmaniose visceral na região neotropical. Apresenta ampla distribuição geográfica e ocorre com freqüência no peridomicílio ${ }^{1}$ e em áreas de grutas calcárias ${ }^{4}$. Na região estudada, mostrou-se mais numerosa no peridomicílio, seguido pelo intradomicílio e poucos exemplares nos outros ecótopos. Os machos de L. longipalpis capturados nesse estudo apresentam-se com apenas uma mancha no $4^{\circ}$ tergito abdominal. Há indícios de que essa espécie represente um complexo de uma ou mais espécies ${ }^{25}$.

O ecótopo preferido de L. carmelinoi foi o peridomicílio, seguido pelo pomar e pela mata, tendo prevalecido no município de Monte do Carmo. É próxima morfologicamente da L. lenti, sendo comum o encontro dessa última em galinheiros ${ }^{6}{ }^{12}$. Este é o primeiro registro de simpatria para as duas espécies. Trabalho sobre hibridização envolvendo ambas espécies já foi realizado e apesar de ocorrer a cópula e a emersão de adultos em F1, não ocorreu sobrevida de $\mathrm{F}^{2}{ }^{2}$.

Além das espécies vetoras já citadas, foram capturadas também L. flaviscutellata vetora de Leishmania $a^{2}$ azonensis ${ }^{18}{ }^{24}$ e L. shannoni encontrada por vários autores naturalmente infectada com flagelados ${ }^{26}$.

Lutzomyia sp 01 é provavelmente uma espécie nova, próxima a Lutzomyia acanthopharynx Martins Falcão \& Silva tendo sido coletados um total de 10 machos e 20 fêmeas (10 exemplares no peridomicílio e 20 no pomar) estando o material reservado para posterior descrição. Já as fêmeas de $L$. (Psathyromyia) sp são semelhantes a $L$. shannoni e $L$. abonnenci, porém apresentam os dutos individuais das espermatecas mais longos que nessas espécies e também estriados. Esse material encontra-se separado para estudo e novas capturas serão realizadas na área para se encontrar os machos dessa espécie e verificar sua verdadeira identidade taxonômica.

Lustosa et $\mathrm{al}^{13}$ trabalharam em três cidades do Estado de Tocantins, tendo capturado L. whitmani em São Sebastião do Tocantins e em Itacajá, sendo que neste último município coletaram também Lutzomyia gomezi (Nitzulescu). Além dessas duas espécies, mais 15 já foram citadas para este estado. Neste artigo, relata-se pela primeira vez a presença de L. longipalpis, L. carmelinoi, L. longipennis, L. sallesi, L. bourrouli, L. aragaoi, L. flaviscutellata, L. micropyga, L. saulensis, L. bacula, L. oswaldoi, L. campbelli, L. claustrei, $L$. rorotaensis, $L$. tuberculata e $L$. walkeri, além do gênero Brumptomyia que não havia sido ainda registrado para o Estado de Tocantins, representado pelas espécies $B$. avellari e $B$. brumpti. Desta forma, a fauna flebotomínica deste estado fica composta, até o momento, por 35 espécies já descritas.

\section{REFERÊNCIAS BIBLIOGRÁFICAS}

1. Aguiar GM, Medeiros WM, De Marco TS, Santos SC, Gambardella S. Ecologia dos flebotomíneos da Serra do Mar, Itaguaí, Estado do Rio de Janeiro, Brasil. I - A fauna flebotomínica e a prevalência pelo local e tipo de captura (Diptera, Psychodidae, Phlebotominae). Cadernos de Saúde Pública 12: 195-206, 1996.

2. Andrade Filho JD, Carneiro APS, Lima MLN, Santiago RM, Gama MA, Santos CA, Falcão AL, Brazil RP. Flebotomíneos de Timóteo, estado de Minas Gerais, Brasil (Diptera: Psychodidae). Cadernos de Saúde Pública 13: 767-770, 1997.
3. Andrade Filho JD, Lima MLN, Falcão AL, Brazil RP. Sazonalidade dos flebotomíneos (Diptera, Psychodidae) dos arredores da Gruta da Lapinha, município de Lagoa Santa, Minas Gerais, Brasil. Revista Brasileira de Entomologia 42: 93-95, 1998.

4. Andrade Filho JD, Alves JCM, Falcão AL, Brazil RP. Hybridization between Lutzomyia lenti (Mangabeira) and Lutzomyia carmelinoi Ryan, Fraiha, Lainson \& Shaw (Diptera: Psychodidae). In: Resumos do $3^{\text {rd }}$ International Symposium on Phlebotomine Sandflies, Montpellier, França p. P25, 1999. 
5. Azevedo ACR, Rangel EF, Costa EM, David J, Vasconcelos AW, Lopes UG. Natural infection of Lutzomyia (Nyssomyia) whitmani (Antunes \& Coutinho, 1939) by Leishmania of the braziliensis complex in Baturité, Ceará state, Northeast Brazil. Memórias do Instituto Oswaldo Cruz 85: 251, 1990.

6. Brazil RP, Morton IE, Ward RD. Notes on the feeding habits of Lutzomyia ( $M$ whitmani (Diptera: Psychodidae) in Ceará State, Northeast Brazil, Memórias do Instituto Oswaldo Cruz 86: 497498, 1991.

7. Brazil RP, Carneiro VL, Andrade Filho JD, Alves JCM, Falcão AL. The colonization and biology of Lutzomyia lenti (Mangabeira). Anais da Sociedade Entomológica do Brazil 26: 191-193, 1997.

8. Cabanillas MRS, Castellón EG. Distribution of sandflies (Diptera: Psychodidae) on tree-trunks in a non-flooded area of Ducke Forest Reserve, Manaus, AM, Brazil. Memórias do Instituto Oswaldo Cruz. 94: 289-296, 1999.

9. Condino MLF, Sampaio SMP, Henriques LD, Galati EAB, Wanderley DMV, Corrêa FMA. Leishmaniose tegumentar americana: flebotomíneos de área de transmissão no município de Teodoro Sampaio, região sudoeste do Estado de São Paulo, Brasil. Revista da Sociedade Brasileira de Medicina Tropical 31: 355-360, 1998.

10. Dias ES, Falcão AL, Silva JE, Martins AV. Taxonomic studies of the subgenus Helcocyrtomyia. I. Series oswaldoi (Diptera, Psychodidae, Phlebotominae). Memórias do Instituto Oswaldo Cruz. 86: 323-340, 1991.

11. Domingos MF, Carreri-Bruno GC, Ciaravolo Rd, Galati EAB, Wandeley DMV, Corrêa FMA. Leishmaniose tegumentar americana: flebotomíneos de área de transmissão, no município de Pedro de Toledo, região sul do estado de São Paulo, Brasil. Revista da Sociedade Brasileira de Medicina Tropical 31: 425432, 1998

12. Gomes AC, Rabello EX, Galati EAB. Flebotomíneos encontrados em galinheiros experimentais nos estados de São Paulo e Minas Gerais (Brasil) e algumas observações ecológicas. Revista de Saúde Pública 12: 403-407, 1978.

13. Lustosa ES, Naves HAM, Carvalho MESD, Barbosa W. Contribuição para o conhecimento da fauna flebotomínica do estado de Goiás - 1984-1985. Nota prévia I. Revista de Patologia Tropical 15: 7-11, 1986

14. Mayo RC, Casanova C, Mascarini LM, Pignatti MG, Rangel O, Galati EAB, Wanderley DM, Corrêa FMA. Flebotomíneos de área de transmissão de leishmaniose tegumentar americana no município de Itupeva, região sudeste do estado de São Paulo, Brasil. Revista da Sociedade Brasileira de Medicina Tropical 31: 339-345, 1998.

15. Queiroz RG, Vasconcelos IAB, Vasconcelos AW, Pessoa FAC, Souza RN, David JR. Cutaneous Leishmaniasis in Ceara State in northeastern Brazil: incrimination of Lutzomyia whitmani (Diptera: Psychodidae) as a vector of Leishmania braziliensis in
Baturite municipality. American Journal of Tropical Medicine and Hygiene 50: 693-698, 1994.

16. Rangel EF, Lainson R, Souza AA, Ready P, Azevedo ACR. Variation between geographical populations of Lutzomyia (Nyssomyia) whitmani (Antunes \& Coutinho, 1939) sensu lato (Diptera: Psychodidae: Phlebotominae) in Brazil. Memórias do Instituto Oswaldo Cruz 91: 43-50, 1996.

17. Rebêlo JMM, Araújo JAC, Carvalho ML, Barros VLL, Silva FS, Oliveira ST. Flebótomos (Diptera, Phlebotominae) da llha de São Luis, zona do Golfão Maranhense, Brasil. Revista da Sociedade Brasileira de Medicina Tropical 32: 247-253, 1999.

18. Ryan L, Silveira FT, Lainson R, Shaw JJ. Leishmanial infections in Lutzomyia longipalpis and Lu. Antunesi (Diptera: Psychodidae) on the island of Marajó, Pará State, Brazil. Transactions of the Royal Society of Tropical Medicine and Hygiene 78: 547-548, 1984.

19. Ryan L, Lainson R, Shaw JJ. Leishmaniasis in Brazil. XXIV. Natural flagellate infections of sandflies (Diptera: Psychodidae) in Pará State, with particular reference to the role of Psychodopygus wellcomei as the vector of Leishmania braziliensis braziliensis in the Serra dos Carajás. Transactions of the Royal Society of Tropical Medicine and Hygiene 81: 353-359, 1987.

20. Shannon RC. Methods for collecting and feeding mosquitoes in jungle yellow fever studies. American Journal of Tropical Medicine and Hygiene 19: 131-148, 1939.

21. Sherlock IA. Ecological interactions of visceral leishmaniasis in the state of Bahia, Brazil. Memórias do Instituto Oswaldo Cruz 91: 671-683, 1996.

22. Sudia WD, Chamberlain RW. Battery operated light trap, an improved model. Mosquito News 22: 126-129, 1962.

23. Teodoro U, La Salvia Filho V, Lima EM, Misuta NM, Verginase TG, Ferreira ME. American cutaneous leishmaniasis: phlebotomine of the area of transmission in the north of Paraná, Brazil. Revista de Saúde Pública 25: 129-133, 1991.

24. Ward RD, Lainson R Shaw JJ. Further evidence of role of Lutzomyia flaviscutellata (Mangabeira) as the vector of Leishmania mexicana amazonensis in Brazil. Transactions of the Royal Society of Tropical Medicine and Hygiene 67: 608-609, 1973.

25. Ward RD, Ribeiro AL, Ready PD, Murtagh A. Reproductive isolation between different forms of Lutzomyia longipalpis (Lutz \& Neiva), (Diptera: Psychodidae), the vector of Leishmania donovani chagasi Cunha \& Chagas and its significance to kalazar distribution in South America. Memórias do Instituto Oswaldo Cruz 78: 269-280, 1983.

26. Young DG, Duncan MA. Guide to the identification and geographic distribution of Lutzomyia sand flies in Mexico, the West Indies, Central and South America (Diptera: Psychodidae). Gainesville, Florida, 1994 